\title{
A NEW POSITIVE TIME-FREQUENCY DISTRIBUTION
}

\author{
Javier R. Fonollosal and Chrysostomos L. Nikias ${ }^{2}$ \\ 'Department of Signal Theory and Communications, Universitat Politènica de Catalunya, Barcelona 08071, SPAIN \\ fono@tsc.upc.es \\ ${ }^{2}$ Center for Research on Applied Signal Processing, University of Southern California, 3740 McClintock Ave, EEB 400B, \\ Los Angeles CA 90089-2564, USA
}

\begin{abstract}
This article studies the formulation of new members of the Cohen-Posch class of positive time-frequency energy distributions. Members of this class are always positive functionals and satisfy the marginal constraints. Therefore, they can be properly interpreted as distributions. We considered the minimization of cross-entropy measures with respect to different priors or "a priori" distributions and the case of no prior or maximum entropy, and concluded with the necessity to introduce new marginal constraints. New mixed in time and frequency constraints are derived based on a "direction invariance" criterion on the time-frequency plane that are directly related to the Fractional Fourier Transform. The implications of this new constrains in the positive class are analyzed from an information theoretic perspective. We conclude that the new constraints provide enough information to fully determine the resulting distribution and thus, no "a priori" distribution is necessary.
\end{abstract}

\section{INTRODUCTION}

Time-frequency representations (TFR) attempt to indicate the distribution of energy density in the timefrequency plane. As such, they must be positive functionals of the signal and satisfy the minimum requirements for a joint distribution. When TFR are integrated along time or frequency, they must yield the correct marginal distributions, i.e. the spectral density of energy and the instantaneous power respectively. The class of "proper" TFR that satisfy the marginals was first defined by Cohen and Posch [1]. It was proved the existence of an infinite number of distributions belonging to the class and that they could all be parametrized by a

'This work was supported by the National Plan of Spain CICYT, Grant number TTC $92-0800-\mathrm{C} 05-05$

${ }^{2}$ This work was supported by ONR, Grant Number function $\rho(x, y)$. Recently, a member of this class was defined by Loughlin et. al. [2, 3]. It was formulated as the solution to a minimum cross-entropy (MCE) optimization problem subject to the linear constraints given by the fulfillment of the marginal conditions. That is, given a prior estimate, $\hat{P}(t, \omega)>0$, of the unknown distribution, $Q(t, \omega)$, it is obtained as,

$Q(t, \omega)=\underset{\hat{Q}(t, \omega)}{\operatorname{argmin}}\left(\iint \hat{Q}(t, \omega) \log \frac{\hat{Q}(t, \omega)}{\hat{P}(t, \omega)} d t d \omega\right)$ subject to,

$$
\begin{aligned}
& \int Q(t, \omega) d t=|S(\omega)|^{2} \\
& \int Q(t, \omega) d \omega=|S(t)|^{2}
\end{aligned}
$$

The Spectrogram or a combination of Spectrograms was chosen as "a priori" distribution $\hat{P}(t, \omega)$. This choice was justified using arguments like its "excellent cross-term properties" or as being the "only known positive estimate of the non-stationary power spectral density that can serve as a prior estimate to MCE". Simulations showed that the "a priori" estimate played indeed a decisive role in the outcome and such discouraging results were blamed on our ignorance about the higher-order moments of a timefrequency energy density. MCE subject only to the two marginal constraints in general can not improve much over the "a priori" distributions unless signal components are oriented in vertical or horizontal lines in the timefrequency plane. In this case, MCE minimization w.r.t. any prior yields basically the same result since the outcome in primarily driven by the marginal constraints.

In our opinion, the limitations of this procedure lay on its high "orientation" dependency. The classical marginal constraints force a high time and frequency resolution IV-301 


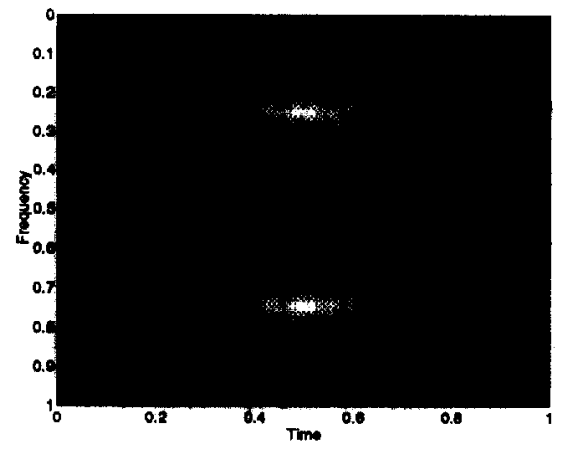

Fig. 1 (a): Spectrogram for two complex exponentials of constant frequency

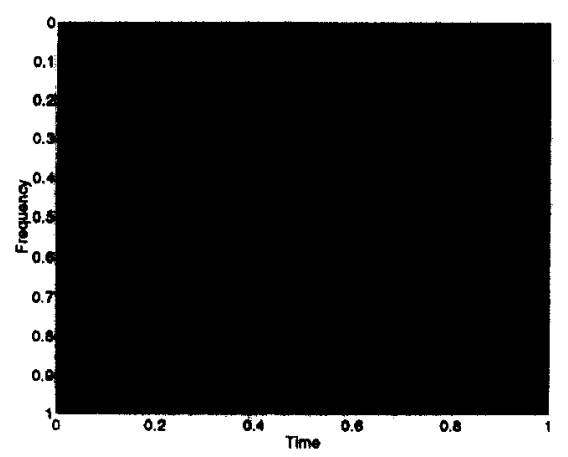

Fig. 1 (b): MCE-TFD for two complex exponentials of constant frequency

along horizontal and vertical lines only, leaving other "orientations" weakly constrained. Let us illustrate this point with an example. Consider two Gaussian envelope complex exponentials of different constant frequency, and a time-frequency "rotated" version of them. The Spectrogram of this signals together with the MCE-TFD with Spectrogram prior are illustrated in Figs. 1-2 (All distributions have been computed with a 256 by 256 resolution). Fig 1 (a) and Fig. 1 (b) for the constant frequencies case and Fig (a) and Fig 2 (b) for the linear frequencies case. The high "orientation" dependency of the MCE-TFD is apparent since a much higher resolution is obtained in the first case compared to the second. New equivalent constraints need to be introduced along nonvertical and non-horizontal directions.

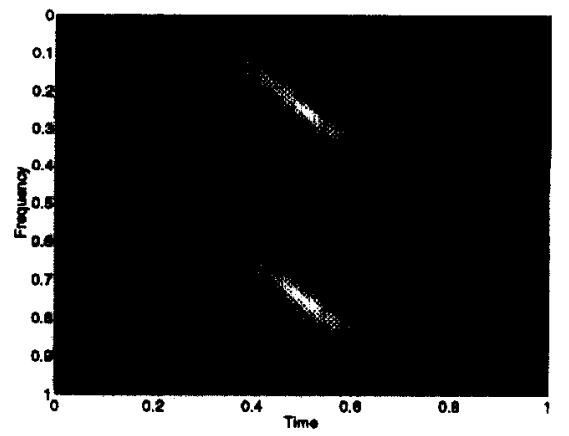

Fig. 2 (a): Spectrogram for two linear chirp signals

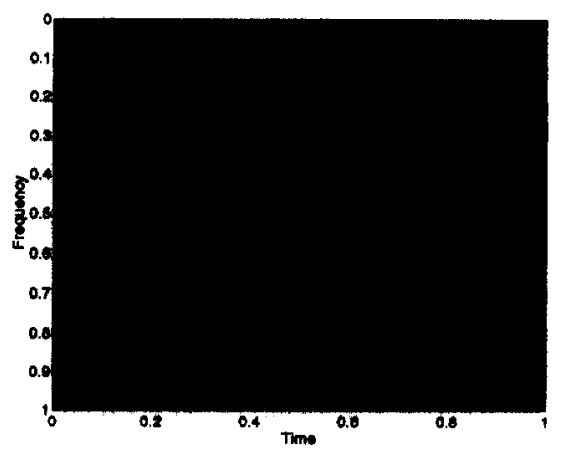

Fig. 2 (b): MCE-TFD for two linear chirp signals

\section{NEW MARGINAL CONSTRAINTS}

The solution, to what the integration of the timefrequency distribution along an arbitrary direction should be, can be found in the Fractional or Angular Fourier Transform (AFT) $[4,5,6$ and references therein]. The AFT decomposes the signal using an orthonormal base of linear chirps, and thus preserves the Parseval relation. The "orientation" of the basis functions (chirps) in the timefrequency plane depends on the angle $\alpha$. Particular cases of the AFT for $\alpha=0$ and $\alpha=\pi / 2$ are the identity operator and the Fourier Transform respectively. Precisely, the AFT of a signal $s(t)$ with angle $\alpha$ is [5],

$$
S_{\alpha}(u)=\int s(t) K_{\alpha}(t, u) d t
$$

where 


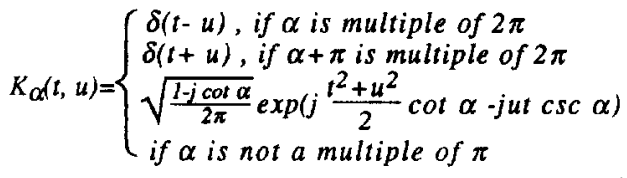

where $\csc$ and cot are the cosecant and cotangent functions respectively. The same way integration of the distribution in the frequency and time axes should give the squared magnitude of the signal and its Fourier transform, as indicated by (2), the integration of the distribution along a rotated axis should give the squared magnitude of the AFT. That is,

$\int_{t} \int_{\omega} P(t, \omega) \delta(t \cos \alpha+\omega \sin \alpha-u) d t d \omega=\left|S_{\alpha}(u)\right|^{2}(5)$

This is a property satisfied by the Wigner-Ville Distribution (WD) [5,6]. One might then be tempted to further constrain the Cohen-Posch class of positive distributions to the subclass that satisfies (5) for all $\alpha$, $-\pi / 2<\alpha \leq \pi / 2$. Unfortunately, (5) can be readily recognized as the Radon Transform [7] of the distribution $P(t, \omega)$ and the Inverse Radon Transform Theorem establishes that a two-dimensional function is uniquely determined from its Radon Transform. Therefore the WD is the only distribution that satisfies (5) and, since the WD is a non-positive functional in general, the subclass of the positive distributions satisfying (5) for all $\alpha$, $-\pi / 2<\alpha<\pi / 2$ is empty.

\section{NEW POSITIVE DISTRIBUTIONS}

Since satisfaction of (5) for all angles is incompatible with positivity, we propose forcing the new marginal constraints for some angles $\alpha_{i}$ only, to restrict the CohenPosch class of positive distributions. From an information theoretic point of view, the two classical marginal constraints, Eq. (2), supply information from the observation of the distribution at angles 0 and $\pi / 2$ only. As seen in the case of MCE-TFD with Spectrogram prior, not much information is provided along other directions and the posterior, the resulting distribution, looks much like the prior when observed from those angles. On the other hand, satisfaction of the generalized marginals (5) at a limited number of angles can restrain the positive class of distributions enough to make the "a priori" approximate distribution unnecessary. Therefore, the new positive

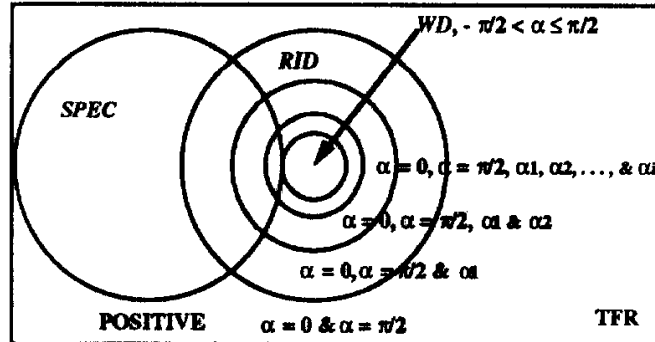

Fig. 3: Effect of the new marginal constraints on TFR.

distribution we propose, ME-TFD, is based on a Maximum Entropy criterion, and is given by,

$Q(t, \omega)=\underset{\hat{Q}(t, \omega)}{\operatorname{argmax}}\left(-\iint \hat{Q}(t, \omega) \log \hat{Q}(t, \omega) d t d \omega\right)(0)$

subject to,

$\iint_{t} Q(t, \omega) \delta(t \cos \alpha+\omega \sin \alpha-u) d t d \omega=\left|S_{\alpha}(u)\right|^{2}(7)$

for $\alpha=0, \alpha=\pi / 2$ and "some" additional $\alpha$. The effect of the constraints on the class of positive distributions is illustrated in Fig. 3.

\section{IMPLEMENTATION}

Resolution of (6) is a multivariate constrained maximization problem that can be solved using the Lagrange multipliers method [8] but demands a huge computational load. Yet, there is an iterative solution to the problem in which each of the univariate optimization problems is solved independently [9]. The same technique was used in [2] for the MCE-TFD. In our case, we start with the uniform distribution (instead of the Spectrogram),

$$
Q_{O}(t, \omega)=\frac{1}{E_{s}} ; E_{s}=\int|s(t)|^{2} d t
$$

and follow the energy normalization iteration,

$$
Q_{k+1}(t, \omega)=\frac{Q_{k}(t, \omega) E_{s}}{\iint_{\omega} Q_{k}(t, \omega) d t d \omega}
$$

and the individual constraint normalizations, 


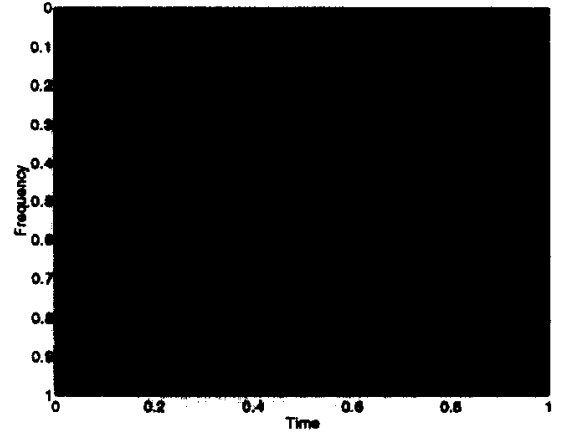

Fig. 4 (a): ME-TFD with the usual constraints only.

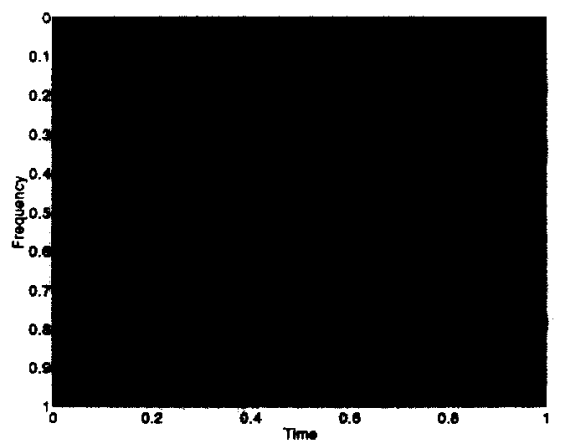

Fig. 4 (b): ME-TFD with two additional constraints.

$Q_{k+1+i}(t, \omega)=$

$\frac{Q_{k+1}(t, \omega)\left|S_{\alpha}(u)\right|^{2}}{\int_{t^{\prime}} \int_{\omega^{\prime}} Q_{k+i}\left(t^{\prime}, \omega^{\prime}\right) \delta\left(t^{\prime} \cos \alpha+\omega^{\prime} \sin \alpha-u\right) d t^{\prime} d \omega^{\prime}}$
for $u=t \cos \alpha+\omega \sin \alpha$

$$
\text { for } u=t \cos \alpha+\omega \sin \alpha
$$

for $\alpha=0, \alpha=\pi / 2$ and the additional $\alpha i$. desired. This iterative algorithm will converge to the optimal distribution provided a solution exists. The number of additional angles chosen will linearly increase the computational complexity. Nevertheless computation of the $A F T$ is not directly required since $\left|S_{\alpha}(u)\right|^{2}$ can be obtained as the integration along the corresponding line of the WD. We consider in our simulations two additional values of $\alpha, \alpha=\pi / 4$ and $\alpha=-\pi / 4$, since in a discrete implementation, these marginals are simply computed as a sum of the diagonals of a matrix. Fig. 3 (a) and Fig 3 (b) show the distributions obtained using ME-TFD with the two usual marginal constraints only and with the addition of the new constraints at angles $\alpha=\pi / 4$ and $\alpha=-\pi / 4$. The "orientation" dependency of the MCE-TFD has been diminished with the addition of only two new constraints

\section{CONCLUSIONS}

A new set of constraints has been proposed for the class of positive time-frequency distributions. They are the equivalent, along non-horizontal or vertical directions, to the two classical marginal conditions and are directly related to the Fractional Fourier Transform. Although the only distribution that satisfies the complete set of new constraints is the WD, they can be partially used to restrain the positive class. A new positive distribution has been presented that derives the maximum entropy distribution subject to the new constraints.

\section{REFERENCES}

[1] Cohen, L. and Posch, T.E. "Positive Time-Frequency Distribution Functions", IEEE Trans. ASSP, vol. ASSP-33, pp. 31-38, 1985.

[2] Loughlin, P.J., Pitton, J.W. and Atlas, L.E., "An Information-Theoretic Approach to Positive TimeFrequency Distributions", Proc of ICASSP-92, San Francisco, CA, pp. V 125-128, 1992.

[3] Pitton, J.W., Loughlin, P.J. and Atlas, L.E., "Positive Time-Frequency ...", Proc of ICASSP-93, Minneapolis, MN, pp. IV 436-439, 1993.

[4] Dickinson, B.W. and Steiglitz, K., "Eigenvectors and Functions of the Discrete Fourier Transform", IEEE Trans. ASSP, vol. ASSP-30, pp. 25-31, 1982.

[5] Almeida, L., "An introduction to the Angular Fourier Transform", Proc of ICASSP-93, Minneapolis, MN, pp. III 257-260, 1993.

[6] Dorsch, R.G. and Lohmann, A.W. "The Fractional Fourier Transform", Proc of ATHOS Workshop on "System Identification and High Order Statistics", Sophia Antipolis, FRANCE, pp. 1-8, 1993.

[7] Deans, S.R., The Radon Transform and Some of Its Applications, New York, Wiley, 1983.

[8] Shore, J.E. and Johnson, R.W. "Properties of CrossEntropy Minimization", IEEE Trans. IT, vol. IT-27, pp. 472-482, 1981.

[9] Alsaka, Y.A., Tzannes, N.S. and Marinelli, W.A., "An efficient algorithm for implementing the relative entropy method", Proc. of ICASSP-88, pp. 23842387,1988 